Research Article

\title{
Exact Solutions for the Integrable Sixth-Order Drinfeld-Sokolov-Satsuma-Hirota System by the Analytical Methods
}

\author{
Jalil Manafian Heris and Mehrdad Lakestani \\ Department of Applied Mathematics, Faculty of Mathematics Science, University of Tabriz, 29 Bahman Boulevard, \\ Tabriz 5166616471, Iran
}

Correspondence should be addressed to Jalil Manafian Heris; j_manafianheris@tabrizu.ac.ir

Received 7 April 2014; Revised 28 May 2014; Accepted 28 May 2014; Published 9 September 2014

Academic Editor: Abdelghani Bellouquid

Copyright (C) 2014 J. Manafian Heris and M. Lakestani. This is an open access article distributed under the Creative Commons Attribution License, which permits unrestricted use, distribution, and reproduction in any medium, provided the original work is properly cited.

\begin{abstract}
We establish exact solutions including periodic wave and solitary wave solutions for the integrable sixth-order Drinfeld-SokolovSatsuma-Hirota system. We employ this system by using a generalized $\left(G^{\prime} / G\right)$-expansion and the generalized tanh-coth methods. These methods are developed for searching exact travelling wave solutions of nonlinear partial differential equations. It is shown that these methods, with the help of symbolic computation, provide a straightforward and powerful mathematical tool for solving nonlinear partial differential equations.
\end{abstract}

\section{Introduction}

The general form of the Drinfeld-Sokolov-Satsuma-Hirota system that is going to be studied in this paper is given by

$$
\begin{gathered}
w_{t}-6 w w_{x}+w_{x x x}-6 v_{x}=0, \\
v_{t}-2 v_{x x x}+6 w v_{x}=0,
\end{gathered}
$$

which was developed in [1] as one example of nonlinear equations possessing Lax pairs of a special form [2-4]. System (1) was independently presented by Drinfeld and Sokolov [1] and Satsuma and Hirota [5]. However, this system was found as a special case of the four-reduction of the KP hierarchy [2, 5]. Wazwaz [6] studied this system by using Hirota's bilinear, the tanh-coth, the tan-cot, and the Exp-function methods. In [2], the truncated singular expansions method was used to construct an explicit Bäcklund transformation method to derive special solutions of this equation. Also, in [3], the sinecosine method and the tanh method were used to obtain exact travelling wave solutions. Recently, the investigation of exact travelling wave solutions to nonlinear partial differential equations plays an important role in the study of nonlinear modelling physical phenomena. The study of the travelling wave solutions plays an important role in nonlinear sciences. Up to now, there exist many powerful methods to construct exact solutions of nonlinear differential equations. A variety of powerful methods have been presented, such as Hirota's bilinear method [7-10], the inverse scattering transform [11], sine-cosine method [12], homotopy perturbation method [13], homotopy analysis method $[14,15]$, variational iteration method [16, 17], Bäcklund transformation [18, 19], Expfunction method $[17,20,21],\left(G^{\prime} / G\right)$-expansion method $[22,23]$, Laplace Adomian decomposition method [24], and differential transform method [25]. Here, we use an effective method for constructing a range of exact solutions for following nonlinear partial differential equations that were first proposed by Wang et al. [26] which a new method called the $\left(G^{\prime} / G\right)$-expansion method to look for travelling wave solutions of NLEEs. Zhang et al. [27] examined the generalized $\left(G^{\prime} / G\right)$-expansion method and its applications. Authors of [28] used $\mathrm{mKdV}$ equation with variable coefficients using the $\left(G^{\prime} / G\right)$-expansion method. Also, Bekir [23] used application of the $\left(G^{\prime} / G\right)$-expansion method for nonlinear evolution equations. In this paper we explain the method which is called the $\left(G^{\prime} / G\right)$-expansion method to look for travelling wave solutions of nonlinear evolution equations. 
The paper is organized as follows. In Section 2, we briefly give the steps of the methods and apply the methods to solve the nonlinear partial differential equations. In Section 3 the application of generalized tanh-coth method to the DSSH equation will be introduced briefly, respectively. Also a conclusion is given in Section 4. Finally some references are given at the end of this paper.

\section{Basic Ideas of the $\left(G^{\prime} / G\right)$-Expansion and the Tanh-Coth Methods}

2.1. The $\left(G^{\prime} / G\right)$-Expansion Method. Another powerful analytical method is called $\left(G^{\prime} / G\right)$-expansion method; we give the detailed description of the method which was first presented by Wang et al. [26].

Step 1. For a given NLPDE with independent variables $X=$ $(x, y, z, t)$ and dependent variable $u$,

$$
\begin{gathered}
\mathscr{P}\left(u, u_{t}, u_{x}, u_{y}, u_{z}, u_{x x}, u_{y y}, u_{z z}, u_{x y},\right. \\
\left.u_{t t}, u_{t x}, u_{t y}, u_{t z}, \ldots\right)=0
\end{gathered}
$$

can be converted to ODE:

$$
\mathscr{M}\left(u,-c u^{\prime}, u^{\prime}, u^{\prime}, u^{\prime \prime}, \ldots\right)=0,
$$

where transformation $\xi=x+y-c t$ is wave variable. Also, $c$ is a constant to be determined later.

Step 2. We seek its solutions in the more general polynomial form as follows:

$$
u(\xi)=a_{0}+\sum_{k=1}^{m} a_{k}\left(\frac{G^{\prime}(\xi)}{G(\xi)}\right)^{k},
$$

where $G(\xi)$ satisfies the second order LODE in the following form:

$$
G^{\prime \prime}(\xi)+\lambda G^{\prime}(\xi)+\mu G(\xi)=0,
$$

where $a_{0}, a_{k}(k=1,2, \ldots, m), \lambda$, and $\mu$ are constants which will be determined later, $a_{m}=0$, but the degree of which is generally equal to or less than $m-1$; the positive integer $m$ can be determined by considering the homogeneous balance between the highest order derivatives and nonlinear terms appearing in (3).

Step 3. Substituting (4) and (5) into (3) with the value of $n$ obtained in Step 1, collecting the coefficients of $\left(G^{\prime}(\xi) / G(\xi)\right)^{k}$ $(k=0,1,2, \ldots)$, and then setting each coefficient to zero, we can get a set of overdetermined partial differential equations for $a_{0}, a_{i}(i=1,2, \ldots, n), \lambda, c$, and $\mu$ with the aid of symbolic computation Maple.

Step 4. Solving the algebraic equations in Step 3 and then substituting $a_{i}, \ldots, a_{m}, c$ and general solutions of (5) into (4) we can obtain a series of fundamental solutions of (2) depending of the solution $G(\xi)$ of (5).

2.2. The Generalized Tanh-Coth Method. We now describe the generalized tanh-coth method for the given partial differential equations. We give the detailed description of the method which to use this method; we take the following steps.
Step 1. For a given NLPDE with independent variables $X=$ $(x, y, z, t)$ and dependent variable $u$, we consider a general form of nonlinear equation

$$
\begin{array}{r}
\mathscr{P}\left(u, u_{t}, u_{x}, u_{y}, u_{z}, u_{x x}, u_{y y}, u_{z z},\right. \\
\left.u_{x y}, u_{t t}, u_{t x}, u_{t y}, u_{t z}, \ldots\right)=0,
\end{array}
$$

which can be converted to ODE

$$
Q\left(u,-c u^{\prime}, u^{\prime}, u^{\prime}, u^{\prime}, u^{\prime \prime}, \ldots\right)=0,
$$

where transformation $\xi=x+y-c t$ is wave variable. Also, $c$ is constant to be determined later.

Step 2. We introduce the Riccati equation as follows:

$$
\Phi^{\prime}=r+p \Phi+q \Phi^{2}, \quad \Phi=\Phi(\xi), \quad \xi=x-c t
$$

which leads to the change of derivatives

$$
\begin{aligned}
\frac{d}{d \xi}= & \left(r+p \Phi+q \Phi^{2}\right) \frac{d}{d \Phi}, \\
\frac{d^{2}}{d \xi^{2}}= & \left(r+p \Phi+q \Phi^{2}\right) \\
& \times\left[(p+2 q \Phi) \frac{d}{d \Phi}+\left(r+p \Phi+q \Phi^{2}\right) \frac{d^{2}}{d \Phi^{2}}\right], \\
\frac{d^{3}}{d \xi^{3}}= & \left(r+p \Phi+q \Phi^{2}\right) \\
& \times\left[\left(6 q^{2} \Phi^{2}+6 p q \Phi+2 r q+p^{2}\right) \frac{d}{d \Phi}\right. \\
& +\left(6 q^{2} \Phi^{3}+9 p q \Phi^{2}+3\left(p^{2}+2 r q\right) \Phi+3 r p\right) \\
& \left.\times \frac{d^{2}}{d \Phi^{2}}+\left(r+p \Phi+q \Phi^{2}\right)^{2} \frac{d^{3}}{d \Phi^{3}}\right],
\end{aligned}
$$

which admits the use of a finite series of functions of the form

$$
u(\xi)=S(\Phi)=\sum_{k=0}^{m} a_{k} \Phi^{k}+\sum_{k=1}^{m} b_{k} \Phi^{-k},
$$

where $a_{k}(k=0,2, \ldots, m), b_{k}(k=1,2, \ldots, m), p, r$, and $q$ are constants to be determined later. But, the positive integer $m$ can be determined by considering the homogeneous balance between the highest order derivatives and nonlinear terms appearing in (7). If $m$ is not an integer, then a transformation formula should be used to overcome this difficulty. For aforementioned method, expansion (10) reduces to the standard tanh method [29] for $b_{k}=0,1 \leq k \leq m$.

Step 3. Substituting (8) and (9) into (7) with the value of $m$ obtained in Step 2, collecting the coefficients of $\Phi^{k}(k=0,1$, $2, \ldots)$, and then setting each coefficient to zero, we can get a set of overdetermined partial differential equations for $a_{0}, a_{i}$ $(i=1,2, \ldots, m), b_{i}(i=1,2, \ldots, m) p, q$, and $r$ with the aid of symbolic computation Maple. 
Step 4. Solve the algebraic equations in Step 3 and then substitute $a_{0}, a_{1}, b_{1}, \ldots, a_{m}, b_{m}, c$ in (10).

Step 5. We will consider that the following twenty seven solutions of generalized Riccati differential equation (8) are given in $[30-32]$.

Case 1. For each $p q \neq 0$ or $q r \neq 0$ and $\Delta=p^{2}-4 q r>0$, (8) has the following solutions:

$$
\begin{aligned}
& \Phi_{1}(\xi)=\frac{-1}{2 q}\left[p+\sqrt{\Delta} \tanh \left(\frac{\sqrt{\Delta} \xi}{2}\right)\right], \\
& \Phi_{2}(\xi)=\frac{-1}{2 q}\left[p+\sqrt{\Delta} \operatorname{coth}\left(\frac{\sqrt{\Delta} \xi}{2}\right)\right], \\
& \Phi_{3}(\xi)=\frac{-1}{2 q}[p+\sqrt{\Delta}[\tanh (\sqrt{\Delta} \xi) \pm i \operatorname{sech}(\sqrt{\Delta} \xi)]] \\
& \Phi_{4}(\xi)=\frac{-1}{2 q}[p+\sqrt{\Delta}[\operatorname{coth}(\sqrt{\Delta} \xi) \pm \operatorname{csch}(\sqrt{\Delta} \xi)]] \\
& \Phi_{5}(\xi)=\frac{-1}{4 q}\left[2 p+\sqrt{-1}\left[\tanh \left(\frac{\sqrt{\Delta} \xi}{4}\right) \pm \operatorname{coth}\left(\frac{\sqrt{\Delta} \xi}{4}\right)\right]\right. \\
& \Phi_{6}(\xi)=\frac{1}{2 q}\left[-p+\frac{\sqrt{\left(A^{2}+B^{2}\right) \Delta}-A \sqrt{\Delta} \cosh (\sqrt{\Delta} \xi)}{A \sinh (\sqrt{\Delta} \xi)+B}\right] \\
& \Phi_{7}(\xi)=\frac{1}{2 q}\left[\begin{array}{c}
\left.-p-\frac{\sqrt{\left(B^{2}-A^{2}\right) \Delta}+A \sqrt{\Delta} \cosh (\sqrt{\Delta} \xi)}{A \sinh (\sqrt{\Delta} \xi)+B}\right] \\
-
\end{array}\right]
\end{aligned}
$$

where $A$ and $B$ are two nonzero real constants and satisfy $B^{2}-$ $A^{2}>0$ :

$$
\begin{aligned}
\Phi_{8}(\xi)= & \frac{2 r \cosh (\sqrt{\Delta} \xi / 2)}{\sqrt{\Delta} \sinh (\sqrt{\Delta} \xi / 2)-p \cosh (\sqrt{\Delta} \xi / 2)}, \\
\Phi_{9}(\xi)= & \frac{-2 r \sinh (\sqrt{\Delta} \xi / 2)}{p \sinh (\sqrt{\Delta} \xi / 2)-\sqrt{\Delta} \cosh (\sqrt{\Delta} \xi / 2)} \\
\Phi_{10}(\xi)= & \frac{2 r \cosh (\sqrt{\Delta} \xi / 2)}{\sqrt{\Delta} \sinh (\sqrt{\Delta} \xi)-p \cosh (\sqrt{\Delta} \xi) \pm i \sqrt{\Delta}} \\
\Phi_{11}(\xi)= & \frac{2 r \sinh (\sqrt{\Delta} \xi / 2)}{-p \sinh (\sqrt{\Delta} \xi)+\sqrt{\Delta} \cosh (\sqrt{\Delta} \xi) \pm \sqrt{\Delta}} \\
\Phi_{12}(\xi)= & (4 r \sinh (\sqrt{\Delta} \xi / 4) \cosh (\sqrt{\Delta} \xi / 4)) \\
& \times(-2 p \sinh (\sqrt{\Delta} \xi / 4) \cosh (\sqrt{\Delta} \xi / 4) \\
& +2 \sqrt{\Delta} \cosh (\sqrt{\Delta} \xi / 4)-\sqrt{\Delta})^{-1} .
\end{aligned}
$$

Case 2. For each $p q \neq 0$ or $q r \neq 0$ and $\Delta=p^{2}-4 q r<0$, (8) has the following solutions:

$$
\begin{aligned}
& \Phi_{13}(\xi)=\frac{-1}{2 q}\left[p-\sqrt{-\Delta} \tan \left(\frac{\sqrt{-\Delta} \xi}{2}\right)\right] \text {, } \\
& \Phi_{14}(\xi)=\frac{-1}{2 q}\left[p+\sqrt{-\Delta} \cot \left(\frac{\sqrt{-\Delta} \xi}{2}\right)\right] \\
& \Phi_{15}(\xi)=\frac{-1}{2 q}[p-\sqrt{-\Delta}[\tan (\sqrt{-\Delta} \xi) \pm \sec (\sqrt{-\Delta} \xi)]] \text {, } \\
& \Phi_{16}(\xi)=\frac{-1}{2 q}[p+\sqrt{-\Delta}[\cot (\sqrt{-\Delta} \xi) \pm \csc (\sqrt{-\Delta} \xi)]] \\
& \Phi_{17}(\xi) \\
& =\frac{-1}{4 q}\left[2 p-\sqrt{-\Delta}\left[\tan \left(\frac{\sqrt{-\Delta} \xi}{4}\right)-\cot \left(\frac{\sqrt{-\Delta} \xi}{4}\right)\right]\right], \\
& \Phi_{18}(\xi) \\
& =\frac{1}{2 q}\left[-p+\frac{ \pm \sqrt{\left(A^{2}+B^{2}\right) \Delta}-A \sqrt{-\Delta} \cos (\sqrt{-\Delta} \xi)}{A \sin (\sqrt{-\Delta} \xi)+B}\right], \\
& \Phi_{19}(\xi) \\
& =\frac{1}{2 q}\left[-p-\frac{ \pm \sqrt{\left(B^{2}-A^{2}\right) \Delta}+A \sqrt{-\Delta} \cos (\sqrt{-\Delta} \xi)}{A \sin (\sqrt{-\Delta} \xi)+B}\right],
\end{aligned}
$$

where $A$ and $B$ are two nonzero real constants and satisfy $B^{2}-$ $A^{2}>0$ :

$$
\begin{aligned}
\Phi_{20}(\xi)= & \frac{-2 r \cos (\sqrt{-\Delta} \xi / 2)}{\sqrt{-\Delta} \sin (\sqrt{-\Delta} \xi / 2)+p \cos (\sqrt{-\Delta} \xi / 2)}, \\
\Phi_{21}(\xi)= & \frac{2 r \sin (\sqrt{-\Delta} \xi / 2)}{-p \sin (\sqrt{-\Delta} \xi / 2)+\sqrt{-\Delta} \cos (\sqrt{-\Delta} \xi / 2)}, \\
\Phi_{22}(\xi)= & \frac{-2 r \cos (\sqrt{-\Delta} \xi / 2)}{\sqrt{-\Delta} \sin (\sqrt{-\Delta} \xi)+p \cos (\sqrt{-\Delta} \xi) \pm \sqrt{-\Delta}}, \\
\Phi_{23}(\xi)= & \frac{2 r \sin (\sqrt{-\Delta} \xi / 2)}{-p \sin (\sqrt{-\Delta} \xi)+\sqrt{-\Delta} \cos (\sqrt{-\Delta} \xi) \pm \sqrt{-\Delta}} \\
\Phi_{24}(\xi)= & (4 r \sin (\sqrt{-\Delta} \xi / 4) \cosh (\sqrt{-\Delta} \xi / 4)) \\
& \times(-2 p \sin (\sqrt{-\Delta} \xi / 4) \cos (\sqrt{-\Delta} \xi / 4) \\
& \left.\quad+2 \sqrt{-\Delta} \cos ^{2}(\sqrt{-\Delta} \xi / 4)-\sqrt{-\Delta}\right)^{-1} .
\end{aligned}
$$


Case 3. For $r=0$ and $p q \neq 0$ (8) has the following solutions:

$$
\begin{aligned}
\Phi_{25}(\xi) & =\frac{-p d}{q[d+\cosh (p \xi)-\sinh (p \xi)]}, \\
\Phi_{26}(\xi) & =\frac{p[\cosh (p \xi)+\sinh (p \xi)]}{q[d+\cosh (p \xi)+\sinh (p \xi)]},
\end{aligned}
$$

where $d$ is an arbitrary constant.

Case 4. For $r=p=0$ and $q \neq 0$ (8) has the following solution:

$$
\Phi_{27}(\xi)=\frac{-1}{q \xi+c},
$$

where $c$ is an arbitrary constant.

But from $\left(G^{\prime} / G\right)$-expansion method, we have

$$
\begin{aligned}
\left(\frac{G^{\prime}}{G}\right)^{\prime} & =\frac{G^{\prime \prime} G-G^{\prime 2}}{G^{2}}=\frac{\left(-\lambda G^{\prime}-\mu G\right) G-G^{\prime 2}}{G^{2}} \\
& =-\mu-\lambda\left(\frac{G^{\prime}}{G}\right)-\left(\frac{G^{\prime}}{G}\right)^{2} ;
\end{aligned}
$$

we set $F=G^{\prime} / G$ and then

$$
F^{\prime}=-\mu-\lambda F-F^{2}
$$

Thus we obtain that the exact solutions derived by $\left(G^{\prime} / G\right)$ expansion are same as ones by the generalized tanh-coth methods. Hence we use only the generalized tanh-coth method.

\section{Application of the Generalized Tanh-Coth Method}

We next consider DSSH equation with the generalized tanhcoth method in the following form:

$$
\begin{gathered}
w_{t}-6 w w_{x}+w_{x x x}-6 v_{x}=0, \\
v_{t}-2 v_{x x x}+6 w v_{x}=0 .
\end{gathered}
$$

Proceeding as before we get

$$
2 u^{(v)}-18 u^{\prime} u^{\prime \prime \prime}-9\left(u^{\prime \prime}\right)^{2}+12\left(u^{\prime}\right)^{3}-c^{2} u^{\prime}-c u^{\prime \prime \prime}=0 .
$$

In order to determine value of $m$, we balance $u^{(v)}$ with $u^{\prime} u^{\prime \prime \prime}$ in (20), and by using (10) we obtain $m=1$. We can suppose that the solutions of (19) are of the following form:

$$
u(\xi)=a_{0}+a_{1} \Phi+\frac{b_{1}}{\Phi} .
$$

Substituting (21) into (20) and by using the well-known software Maple, we obtain the system of the following results:

$$
a_{0}=2 q, \quad a_{1}=2 q, \quad b_{1}=0, \quad c=p^{2}-4 q r,
$$

or

$$
a_{0}=2 r, \quad a_{1}=0, \quad b_{1}=-2 r, \quad c=p^{2}-4 q r,
$$

where $p, q, r$, and $c$ are arbitrary constants. Substituting (22) and (23) into expression (21) we obtain

$$
\begin{array}{ll}
u(\xi)=2 q+2 q \Phi(\xi), & \xi=x-\left(p^{2}-4 q r\right) t \\
u(\xi)=2 r-\frac{2 r}{\Phi(\xi)}, & \xi=x-\left(p^{2}-4 q r\right) t
\end{array}
$$

By the manipulation as explained in the previous section, we have the following.

(I) The First Set for (24). By using Case 1 from Section 2 we have

$$
\begin{aligned}
& u_{1}(x, t)=2 q\left\{1-\frac{1}{2 q}\left[p+\sqrt{\Delta} \tanh \left(\frac{\sqrt{\Delta} \xi}{2}\right)\right]\right\}, \\
& u_{2}(x, t)=2 q\left\{1-\frac{1}{2 q}\left[p+\sqrt{\Delta} \operatorname{coth}\left(\frac{\sqrt{\Delta} \xi}{2}\right)\right]\right\},
\end{aligned}
$$

$u_{3}(x, t)$

$$
=2 q\left\{1-\frac{1}{2 q}[p+\sqrt{\Delta}[\tanh (\sqrt{\Delta} \xi) \pm i \operatorname{sech}(\sqrt{\Delta} \xi)]]\right\},
$$

$u_{4}(x, t)$

$$
\begin{gathered}
=2 q\left\{1-\frac{1}{2 q}[p+\sqrt{\Delta}[\operatorname{coth}(\sqrt{\Delta} \xi) \pm \operatorname{csch}(\sqrt{\Delta} \xi)]]\right\}, \\
u_{5}(x, t) \\
=2 q\left\{1-\frac{1}{4 q} \quad\left[2 p+\sqrt{\Delta}\left[\tanh \left(\frac{\sqrt{\Delta} \xi}{4}\right)\right.\right.\right. \\
\left.\left.\left.\times\left[\frac{\sqrt{\Delta} \xi}{4}\right)\right]\right]\right\},
\end{gathered}
$$

$u_{6}(x, t)$

$=2 q\left\{1+\frac{1}{2 q}\left[-p+\frac{\sqrt{\left(A^{2}+B^{2}\right) \Delta}-A \sqrt{\Delta} \cosh (\sqrt{\Delta} \xi)}{A \sinh (\sqrt{\Delta} \xi)+B}\right]\right\}$,

$u_{7}(x, t)$

$=2 q\left\{1+\frac{1}{2 q}\left[-p-\frac{\sqrt{\left(B^{2}-A^{2}\right) \Delta}+A \sqrt{\Delta} \cosh (\sqrt{\Delta} \xi)}{A \sinh (\sqrt{\Delta} \xi)+B}\right]\right\}$, 


$$
\begin{aligned}
& u_{8}(x, t) \\
& =2 q\left\{1+\frac{2 r \cosh (\sqrt{\Delta} \xi / 2)}{\sqrt{\Delta} \sinh (\sqrt{\Delta} \xi / 2)-p \cosh (\sqrt{\Delta} \xi / 2)}\right\}, \\
& u_{9}(x, t) \\
& =2 q\left\{1+\frac{-2 r \sinh (\sqrt{\Delta} \xi / 2)}{p \sinh (\sqrt{\Delta} \xi / 2)-\sqrt{\Delta} \cosh (\sqrt{\Delta} \xi / 2)}\right\}, \\
& u_{10}(x, t) \quad 2 q\left\{1+\frac{2 r \cosh (\sqrt{\Delta} \xi / 2)}{\sqrt{\Delta} \sinh (\sqrt{\Delta} \xi)-p \cosh (\sqrt{\Delta} \xi) \pm i \sqrt{\Delta}}\right\}, \\
& u_{11}(x, t) \quad 2 q\left\{1+\frac{2 r \sinh (\sqrt{\Delta} \xi / 2)}{-p \sinh (\sqrt{\Delta} \xi)+\sqrt{\Delta} \cosh (\sqrt{\Delta} \xi) \pm \sqrt{\Delta}}\right\}, \\
& u_{12}(x, t)=2 q\{1+(4 r \sinh (\sqrt{\Delta} \xi / 4) \cosh (\sqrt{\Delta} \xi / 4)) \\
& \times(-2 p \sinh (\sqrt{\Delta} \xi / 4) \cosh (\sqrt{\Delta} \xi / 4) \\
& \left.\left.+2 \sqrt{\Delta} \cosh { }^{2}(\sqrt{\Delta} \xi / 4)-\sqrt{\Delta}\right)^{-1}\right\} .
\end{aligned}
$$

By using Case 2 from Section 2 we have

$$
\begin{aligned}
& u_{13}(x, t)=2 q\left\{1-\frac{1}{2 q}\left[p-\sqrt{-\Delta} \tan \left(\frac{\sqrt{-\Delta} \xi}{2}\right)\right]\right\}, \\
& u_{14}(x, t)=2 q\left\{1-\frac{1}{2 q}\left[p+\sqrt{-\Delta} \cot \left(\frac{\sqrt{-\Delta} \xi}{2}\right)\right]\right\},
\end{aligned}
$$$$
u_{15}(x, t)
$$$$
=2 q\left\{1-\frac{1}{2 q}[p-\sqrt{-\Delta}[\tan (\sqrt{-\Delta} \xi) \pm \sec (\sqrt{-\Delta} \xi)]]\right\},
$$$$
u_{16}(x, t)
$$$$
=2 q\left\{1-\frac{1}{2 q}[p+\sqrt{-\Delta}[\cot (\sqrt{-\Delta} \xi) \pm \csc (\sqrt{-\Delta} \xi)]]\right\},
$$$$
u_{17}(x, t)
$$$$
=2 q\left\{1-\frac{1}{4 q}\right.
$$$$
\times\left[2 p-\sqrt{-\Delta}\left[\tan \left(\frac{\sqrt{-\Delta} \xi}{4}\right)\right.\right.
$$$$
\left.\left.\left.-\cot \left(\frac{\sqrt{-\Delta} \xi}{4}\right)\right]\right]\right\},
$$

$u_{18}(x, t)$

$$
\begin{aligned}
& =2 q\left\{1+\frac{1}{2 q}\right. \\
& \times\left[-p+\left( \pm \sqrt{\left(A^{2}+B^{2}\right) \Delta}-A \sqrt{-\Delta} \cos (\sqrt{-\Delta} \xi)\right)\right. \\
& \left.\left.\times(A \sin (\sqrt{-\Delta} \xi)+B)^{-1}\right]\right\}, \\
& u_{19}(x, t) \\
& =2 q\left\{1+\frac{1}{2 q}\right. \\
& \times\left[-p-\left( \pm \sqrt{\left(B^{2}-A^{2}\right) \Delta}+A \sqrt{-\Delta} \cos (\sqrt{-\Delta} \xi)\right)\right. \\
& \left.\left.\times(A \sin (\sqrt{-\Delta} \xi)+B)^{-1}\right]\right\}, \\
& u_{20}(x, t) \\
& =2 q\left\{1+\frac{-2 r \cos (\sqrt{-\Delta} \xi / 2)}{\sqrt{-\Delta} \sin (\sqrt{-\Delta} \xi / 2)+p \cos (\sqrt{-\Delta} \xi / 2)}\right\},
\end{aligned}
$$$$
u_{21}(x, t)
$$$$
=2 q\left\{1+\frac{2 r \sin (\sqrt{-\Delta} \xi / 2)}{-p \sin (\sqrt{-\Delta} \xi / 2)+\sqrt{-\Delta} \cos (\sqrt{-\Delta} \xi / 2)}\right\},
$$

$u_{22}(x, t)$

$$
\begin{aligned}
& =2 q\left\{1+\frac{-2 r \cos (\sqrt{-\Delta} \xi / 2)}{\sqrt{-\Delta} \sin (\sqrt{-\Delta} \xi)+p \cos (\sqrt{-\Delta} \xi) \pm \sqrt{-\Delta}}\right\}, \\
& u_{23}(x, t) \\
& =2 q\left\{1+\frac{2 r \sin (\sqrt{-\Delta} \xi / 2)}{-p \sin (\sqrt{-\Delta} \xi)+\sqrt{-\Delta} \cos (\sqrt{-\Delta} \xi) \pm \sqrt{-\Delta}}\right\},
\end{aligned}
$$

$$
\begin{aligned}
& u_{24}(x, t) \\
& =2 q\left\{1+\left(4 r \sin \left(\frac{\sqrt{-\Delta} \xi}{4}\right) \cosh \left(\frac{\sqrt{-\Delta} \xi}{4}\right)\right)\right. \\
& \times\left(-2 p \sin \left(\frac{\sqrt{-\Delta} \xi}{4}\right) \cos \left(\frac{\sqrt{-\Delta} \xi}{4}\right)\right. \\
& \left.\left.+2 \sqrt{-\Delta} \cos ^{2}\left(\frac{\sqrt{-\Delta} \xi}{4}\right)-\sqrt{-\Delta}\right)^{-1}\right\} \text {. }
\end{aligned}
$$

By using Case 3 from Section 2 we have

$$
\begin{aligned}
& u_{25}(x, t)=2 q\left\{1+\frac{-p d}{q[d+\cosh (p \xi)-\sinh (p \xi)]}\right\}, \\
& u_{26}(x, t)=2 q\left\{1+\frac{p[\cosh (p \xi)+\sinh (p \xi)]}{q[d+\cosh (p \xi)+\sinh (p \xi)]}\right\} .
\end{aligned}
$$


By using Case 4 from Section 2 we have

$$
u_{27}(x, t)=2 q\left\{1-\frac{1}{q \xi+c}\right\}
$$

where $\xi=x-\left(p^{2}-4 q r\right) t$.

(II) The Second Set for (25). By using Case 1 from Section 2 we have

$$
\begin{aligned}
& u_{1}(x, t)=2 r\left\{1+\frac{2 q}{[p+\sqrt{\Delta} \tanh (\sqrt{\Delta} \xi / 2)]}\right\}, \\
& u_{2}(x, t)=2 r\left\{1+\frac{2 q}{[p+\sqrt{\Delta} \operatorname{coth}(\sqrt{\Delta} \xi / 2)]}\right\}, \\
& u_{3}(x, t) \\
& =2 r\left\{1+\frac{2 q}{[p+\sqrt{\Delta}[\tanh (\sqrt{\Delta} \xi) \pm i \operatorname{sech}(\sqrt{\Delta} \xi)]]},\right. \\
& u_{4}(x, t) \\
& =2 r\left\{1+\frac{[p+\sqrt{\Delta}[\operatorname{coth}(\sqrt{\Delta} \xi) \pm \operatorname{csch}(\sqrt{\Delta} \xi)]]}{u_{5}(x, t)}\right. \\
& =2 r\{1+4 q \\
& \times\left[2 p+\sqrt{\Delta}\left[\tanh \left(\frac{\sqrt{\Delta} \xi}{4}\right)\right.\right. \\
& \left.\left.\quad\left\{\operatorname{coth}\left(\frac{\sqrt{\Delta} \xi}{4}\right)\right]\right]^{-1}\right\}
\end{aligned}
$$$$
u_{6}(x, t)
$$$$
=2 r\{1-2 q
$$$$
\times\left[-p+\left(\left(\sqrt{\left(A^{2}+B^{2}\right) \Delta}-A \sqrt{\Delta} \cosh (\sqrt{\Delta} \xi)\right)\right.\right.
$$$$
\left.\left.\left.+(A \sinh (\sqrt{\Delta} \xi)+B)^{-1}\right)\right]^{-1}\right\},
$$

$$
\begin{aligned}
& u_{7}(x, t) \\
& =2 r\{1-2 q \\
& \times\left[-p-\left(\sqrt{\left(B^{2}-A^{2}\right) \Delta}+A \sqrt{\Delta} \cosh (\sqrt{\Delta} \xi)\right)\right. \\
& \left.\left.\times(A \sinh (\sqrt{\Delta} \xi)+B)^{-1}\right]^{-1}\right\},
\end{aligned}
$$

$$
\begin{aligned}
& u_{8}(x, t) \\
& \quad=2 r\left\{1-\frac{\sqrt{\Delta} \sinh (\sqrt{\Delta} \xi / 2)-p \cosh (\sqrt{\Delta} \xi / 2)}{2 r \cosh (\sqrt{\Delta} \xi / 2)}\right\},
\end{aligned}
$$

$u_{9}(x, t)$

$$
=2 r\left\{1+\frac{p \sinh (\sqrt{\Delta} \xi / 2)-\sqrt{\Delta} \cosh (\sqrt{\Delta} \xi / 2)}{2 r \sinh (\sqrt{\Delta} \xi / 2)}\right\},
$$

$$
\begin{aligned}
& u_{10}(x, t) \\
& =2 r\left\{1-\frac{\sqrt{\Delta} \sinh (\sqrt{\Delta} \xi)-p \cosh (\sqrt{\Delta} \xi) \pm i \sqrt{\Delta}}{2 r \cosh (\sqrt{\Delta} \xi / 2)}\right\}, \\
& u_{11}(x, t) \\
& =2 r\left\{1-\frac{-p \sinh (\sqrt{\Delta} \xi)+\sqrt{\Delta} \cosh (\sqrt{\Delta} \xi) \pm \sqrt{\Delta}}{2 r \sinh (\sqrt{\Delta} \xi / 2)}\right\}, \\
& u_{12}(x, t) \\
& =2 r\left\{1-\left(-2 p \sinh \left(\frac{\sqrt{\Delta} \xi}{4}\right) \cosh \left(\frac{\sqrt{\Delta} \xi}{4}\right)\right.\right. \\
& \left.\times\left(4 r \sinh \left(\frac{\sqrt{\Delta} \xi}{4}\right) \cosh \left(\frac{\sqrt{\Delta} \xi}{4}\right)\right)^{-1}\right\} .
\end{aligned}
$$

By using Case 2 from Section 2 we have

$$
u_{13}(x, t)=2 r\left\{1+\frac{2 q}{[p-\sqrt{-\Delta} \tan (\sqrt{-\Delta} \xi / 2)]}\right\},
$$$$
u_{14}(x, t)=2 r\left\{1+\frac{2 q}{[p+\sqrt{-\Delta} \cot (\sqrt{-\Delta} \xi / 2)]}\right\},
$$

$u_{15}(x, t)$

$$
=2 r\left\{1+\frac{2 q}{[p-\sqrt{-\Delta}[\tan (\sqrt{-\Delta} \xi) \pm \sec (\sqrt{-\Delta} \xi)]]}\right\},
$$

$u_{16}(x, t)$

$$
=2 r\left\{1+\frac{2 q}{[p+\sqrt{-\Delta}[\cot (\sqrt{-\Delta} \xi) \pm \csc (\sqrt{-\Delta} \xi)]]}\right\},
$$

$u_{17}(x, t)$

$$
=2 r\left\{1+\frac{4 q}{[2 p-\sqrt{-\Delta}[\tan (\sqrt{-\Delta} \xi / 4)-\cot (\sqrt{-\Delta} \xi / 4)]]}\right\},
$$




$$
\begin{aligned}
& u_{18}(x, t) \\
& =2 r\{1-2 q \\
& \times\left[-p+\left( \pm \sqrt{\left(A^{2}+B^{2}\right) \Delta}-A \sqrt{-\Delta} \cos (\sqrt{-\Delta} \xi)\right)\right. \\
& \left.\left.\times(A \sin (\sqrt{-\Delta} \xi)+B)^{-1}\right]^{-1}\right\}, \\
& u_{19}(x, t) \\
& =2 r\{1-2 q \\
& \times\left[-p-\left( \pm \sqrt{\left(B^{2}-A^{2}\right) \Delta}+A \sqrt{-\Delta} \cos (\sqrt{-\Delta} \xi)\right)\right. \\
& \left.\left.\times(A \sin (\sqrt{-\Delta} \xi)+B)^{-1}\right]^{-1}\right\}, \\
& u_{20}(x, t) \\
& =2 r\left\{1+\frac{\sqrt{-\Delta} \sin (\sqrt{-\Delta} \xi / 2)+p \cos (\sqrt{-\Delta} \xi / 2)}{2 r \cos (\sqrt{-\Delta} \xi / 2)}\right\}, \\
& u_{21}(x, t) \\
& =2 r\left\{1-\frac{-p \sin (\sqrt{-\Delta} \xi / 2)+\sqrt{-\Delta} \cos (\sqrt{-\Delta} \xi / 2)}{2 r \sin (\sqrt{-\Delta} \xi / 2)}\right\}, \\
& u_{22}(x, t) \\
& =2 r\left\{1+\frac{\sqrt{-\Delta} \sin (\sqrt{-\Delta} \xi)+p \cos (\sqrt{-\Delta} \xi) \pm \sqrt{-\Delta}}{2 r \cos (\sqrt{-\Delta} \xi / 2)}\right\}, \\
& u_{23}(x, t) \\
& =2 r\left\{1-\frac{-p \sin (\sqrt{-\Delta} \xi)+\sqrt{-\Delta} \cos (\sqrt{-\Delta} \xi) \pm \sqrt{-\Delta}}{2 r \sin (\sqrt{-\Delta} \xi / 2)}\right\}, \\
& u_{24}(x, t) \\
& =2 r\left\{1-\left(-2 p \sin \left(\frac{\sqrt{-\Delta} \xi}{4}\right) \cos \left(\frac{\sqrt{-\Delta} \xi}{4}\right)\right.\right. \\
& \left.+2 \sqrt{-\Delta} \cos ^{2}\left(\frac{\sqrt{-\Delta} \xi}{4}\right)-\sqrt{-\Delta}\right) \\
& \left.\times\left(4 r \sin \left(\frac{\sqrt{-\Delta} \xi}{4}\right) \cosh \left(\frac{\sqrt{-\Delta} \xi}{4}\right)\right)^{-1}\right\} \text {. }
\end{aligned}
$$

By using Case 3 from Section 2 we have

$$
\begin{aligned}
& u_{25}(x, t)=2 r\left\{1+\frac{q[d+\cosh (p \xi)-\sinh (p \xi)]}{p d}\right\}, \\
& u_{26}(x, t)=2 r\left\{1-\frac{q[d+\cosh (p \xi)+\sinh (p \xi)]}{p[\cosh (p \xi)+\sinh (p \xi)]}\right\}
\end{aligned}
$$

By using Case 4 from Section 2 we have

$$
u_{27}(x, t)=2 r\{1+q \xi+c\},
$$

where $\xi=x-\left(p^{2}-4 q r\right) t$. By using the $w(x, t)=u_{x}(x, t)$, $v(x, t)=(1 / 6)\left(u_{t}-3\left(u_{x}\right)^{2}+u_{x x x}\right)$, can be used to get the solutions of the DSSH system (19). It can be seen that the results are the same, with comparing results in the literature [6]. We obtained analytical solutions by the generalized $\left(G^{\prime} / G\right)$ expansion and the generalized tanh-coth methods. Also, in this paper we can see correlation between $\left(G^{\prime} / G\right)$-expansion method and tanh-coth methods. We have succeeded in identifying the equivalence of the two methods under special conditions [33]. Consequently, the solution of the equations via $\left(G^{\prime} / G\right)$-expansion method is exactly the same as the solution of tanh-coth method if the conditions are satisfied. In fact, we can see that the tanh-coth method is a special case of the $\left(G^{\prime} / G\right)$-expansion method.

\section{Conclusion}

In this paper we investigated the Drinfeld-Sokolov-SatsumaHirota system by using the generalized $\left(G^{\prime} / G\right)$-expansion and the generalized tanh-coth methods which are useful methods for finding travelling wave solutions of nonlinear evolution equations. These methods have been successfully applied to obtain some new generalized solitonary solutions to the DSSH equation. These exact solutions include three types: hyperbolic function solution, trigonometric function solution, and rational solution. The generalized $\left(G^{\prime} / G\right)$ expansion method is more powerful in searching for exact solutions of NLPDEs. By comparing our results and Wazwaz's [6] results it can be seen that the results are the same. Also, new results are formally developed in this paper. It can be concluded that this method is a very powerful and efficient technique in finding exact solutions for wide classes of problems.

\section{Conflict of Interests}

The authors declare that there is no conflict of interests regarding the publication of this paper.

\section{Acknowledgment}

The authors are very grateful to both referees for their comments and suggestions.

\section{References}

[1] V. G. Drinfeld and V. V. Sokolov, "Equations of Korteweg-de Vries type and simple Lie algebras," Doklady Akademii Nauk, vol. 258, pp. 11-16, 1981.

[2] A. Karsau-Kalkani and S. Yu Sarkovich, "Bäcklund transformation and special solutions for Drinfeld-Sokolov-SatsumaHirota system of coupled equations," Journal of Physics A: Mathematical and General, vol. 34, pp. 7353-7358, 2001.

[3] A. M. Wazwaz, "Exact and explicit travelling wave solutions for the nonlinear Drinfeld-Sokolov system," Communications in Nonlinear Science and Numerical Simulation, vol. 11, no. 3, pp. 311-325, 2006 
[4] A. Karsau-Kalkani, A. Karsau, A. Sakovich, S. Sarkovich, and R. Turhan, "A new integrable generalization of the Korteweg-de Vries equation," Journal of Mathematical Physics, vol. 49, pp. 110, 2008.

[5] J. Satsuma and R. Hirota, "A coupled KdV equation is one case of the four-reduction of the KP hierarchy," Journal of the Physical Society of Japan, vol. 51, no. 10, pp. 3390-3397, 1982.

[6] A. M. Wazwaz, "The Cole-Hopf transformation and multiple soliton solutions for the integrable sixth-order DrinfeldSokolov-Satsuma-Hirota equation," Applied Mathematics and Computation, vol. 207, no. 1, pp. 248-255, 2009.

[7] R. Hirota and M. Ito, "Resonance of solitons in one dimension," Journal of the Physical Society of Japan, vol. 52, no. 3, pp. 744$748,1983$.

[8] M. Ito, "An extension of nonlinear evolution equations of the $\mathrm{K}-\mathrm{dV}$ (mK-dV) type to higher orders," Journal of the Physical Society of Japan, vol. 49, no. 2, pp. 771-778, 1980.

[9] J. Hietarinta, "A search for bilinear equations passing Hirota's three-soliton condition. I. KdV-type bilinear equations," Journal of Mathematical Physics, vol. 28, no. 8, pp. 1732-1742, 1987.

[10] W. Hereman and W. Zhuang, "AMACSYMA program for the Hirota method," in Proceedings of the 13th IMACS World Congress on Computation and Applied Mathematics, pp. 22-26, 1991.

[11] M. J. Ablowitz and P. A. Clarkson, Solitons, Nonlinear Evolution Equations and Inverse Scattering, Cambridge University Press, Cambridge, UK, 1991.

[12] A.-M. Wazwaz, "Travelling wave solutions for combined and double combined sine-cosine-Gordon equations by the variable separated ODE method," Applied Mathematics and Computation, vol. 177, no. 2, pp. 755-760, 2006.

[13] M. Dehghan and J. Manafian, "The solution of the variable coefficients fourth-order parabolic partial differential equations by homotopy perturbation method," Zeitschrift für Naturforschung, vol. 64, pp. 420-430, 2009.

[14] M. Dehghan, J. Manafian, and A. Saadatmandi, "The solution of the linear fractional partial differential equations using the homotopy analysis method," Zeitschrift für Naturforschung, vol. 65, no. 11, pp. 935-949, 2010.

[15] M. Dehghan, J. Manafian, and A. Saadatmandi, "Solving nonlinear fractional partial differential equations using the homotopy analysis method," Numerical Methods for Partial Differential Equations, vol. 26, no. 2, pp. 448-479, 2010.

[16] J. H. He, "Variational iteration method-a kind of non-linear analytical technique: Some examples," International Journal of Non-Linear Mechanics, vol. 34, no. 4, pp. 699-708, 1999.

[17] M. Dehghan, J. M. Heris, and A. Saadatmandi, "Application of the Exp-function method for solving a partial differential equation arising in biology and population genetics," International Journal of Numerical Methods for Heat and Fluid Flow, vol. 21, no. 6, pp. 736-753, 2011.

[18] X. H. Menga, W. J. Liua, H. W. Zhua, C. Y. Zhang, and B. Tian, "Multi-soliton solutions and a Bäcklund transformation for a generalized variable-coefficient higher-order nonlinear Schrödinger equation with symbolic computation," Journal of Physics A, vol. 387, no. 97, 107 pages, 2008.

[19] X. Lü, H. W. Zhu, X. H. Meng, Z. C. Yang, and B. Tian, "Soliton solutions and a Bäcklund transformation for a generalized nonlinear Schrödinger equation with variable coefficients from optical fiber communications," Journal of Mathematical Analysis and Applications, vol. 336, no. 2, pp. 1305-1315, 2007.
[20] J. H. He and X. H. Wu, "Exp-function method for nonlinear wave equations," Chaos, Solitons and Fractals, vol. 30, no. 3, pp. 700-708, 2006.

[21] J. Manafian Heris and M. Bagheri, "Exact solutions for the modified $\mathrm{KdV}$ and the generalized $\mathrm{KdV}$ equations via Exp-function method," Journal of Mathematical Extension, vol. 4, pp. 77-98, 2010.

[22] M. Fazli Aghdaei and J. Manafianheris, "Exact solutions of the couple Boiti-Leon-Pempinelli system by the generalized $\left(G^{\prime} / G\right)$-expansion method," Journal of Mathematical Extension, vol. 5, pp. 91-104, 2011.

[23] A. Bekir, "Application of the $\left(G^{\prime} / G\right)$-expansion method for nonlinear evolution equations," Physics Letters A, vol. 372, no. 19, pp. 3400-3406, 2008.

[24] J. Manafianheris, "Solving the integro-differential equations using the modified Laplace Adomian decomposition method," Journal of Mathematical Extension, vol. 6, pp. 41-55, 2012.

[25] M. Bagheri and J. Manafian Heris, "Differential transform method for solving the linear and nonlinear Westervelt equation," Journal of Mathematical Extension, vol. 6, pp. 81-91, 2012.

[26] M. Wang, X. Li, and J. Zhang, “The $\left(G^{\prime} / G\right)$-expansion method and travelling wave solutions of nonlinear evolution equations in mathematical physics," Physics Letters A, vol. 372, no. 4, pp. 417-423, 2008.

[27] J. Zhang, X. Wei, and Y. Lu, "A generalized $\left(G^{\prime} / G\right)$-expansion method and its applications," Physics Letters A, vol. 372, no. 20, pp. 3653-3658, 2008.

[28] S. Zhang, J. L. Tong, and W. Wang, "A generalized $\left(G^{\prime} / G\right)$ expansion method for the $\mathrm{mKdV}$ equation with variable coefficients," Physics Letters A, vol. 372, no. 13, pp. 2254-2257, 2008.

[29] W. Malfliet, "Solitary wave solutions of nonlinear wave equations," The American Journal of Physics, vol. 60, pp. 650-654, 1992.

[30] S. D. Zhu, “The generalizing Riccati equation mapping method in non-linear evolution equation: application to $(2+1)$-dimensional Boiti-Leon-Pempinelle equation," Chaos, Solitons and Fractals, vol. 37, no. 5, pp. 1335-1342, 2008.

[31] Z. Li and X. Zhang, "New exact kink solutions and periodic form solutions for a generalized Zakharov-Kuznetsov equation with variable coefficients," Communications in Nonlinear Science and Numerical Simulation, vol. 15, no. 11, pp. 3418-3422, 2010.

[32] M. E. Zayed Elsayed and H. ARNOUS Ahmed, "Many families of exact solutions for nonlinear system of partial differential equations describing the dynamics of DNA," Journal of Partial Differential Equations, vol. 26, pp. 373-384, 2013.

[33] M. E. Zayed Elsayed, "Equivalence of the $\left(G^{\prime} / G\right)$-expansion method and the Tanh-Coth function method," in Proceedings of the International Conference on Numerical Analysis and Applied Mathematics (ICNAAM '10), pp. 2225-2228, September 2010. 


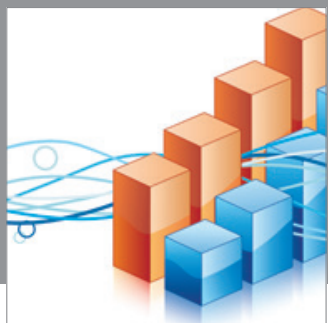

Advances in

Operations Research

mansans

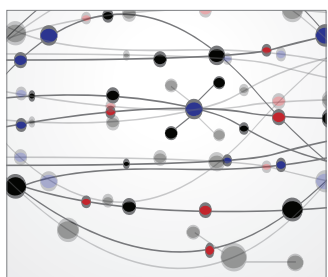

The Scientific World Journal
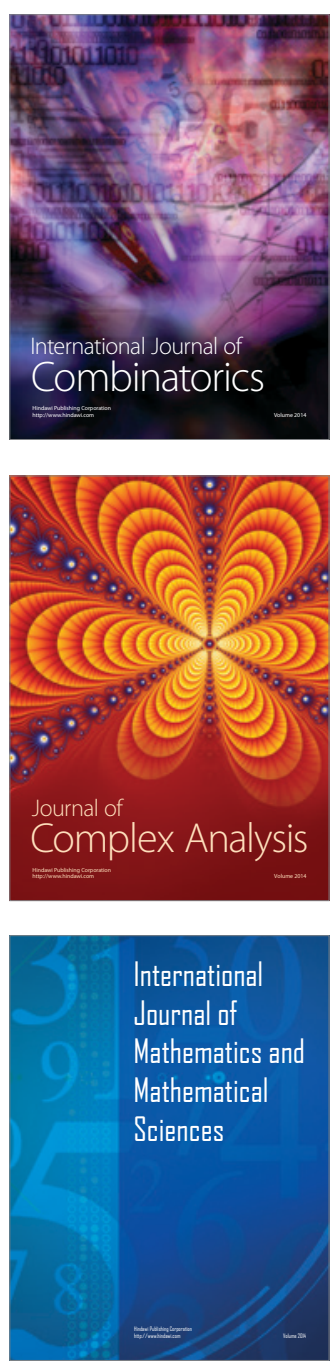
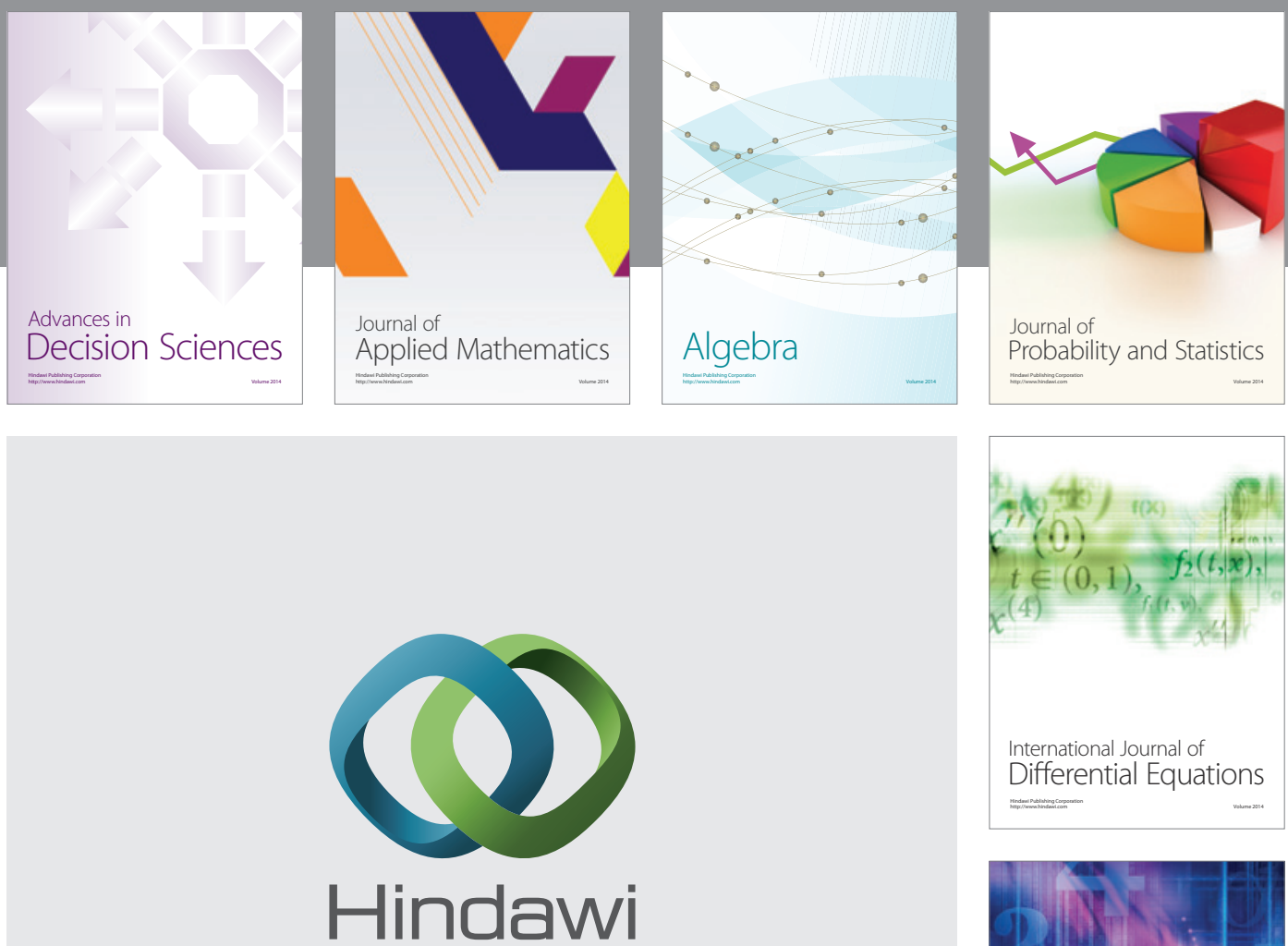

Submit your manuscripts at http://www.hindawi.com
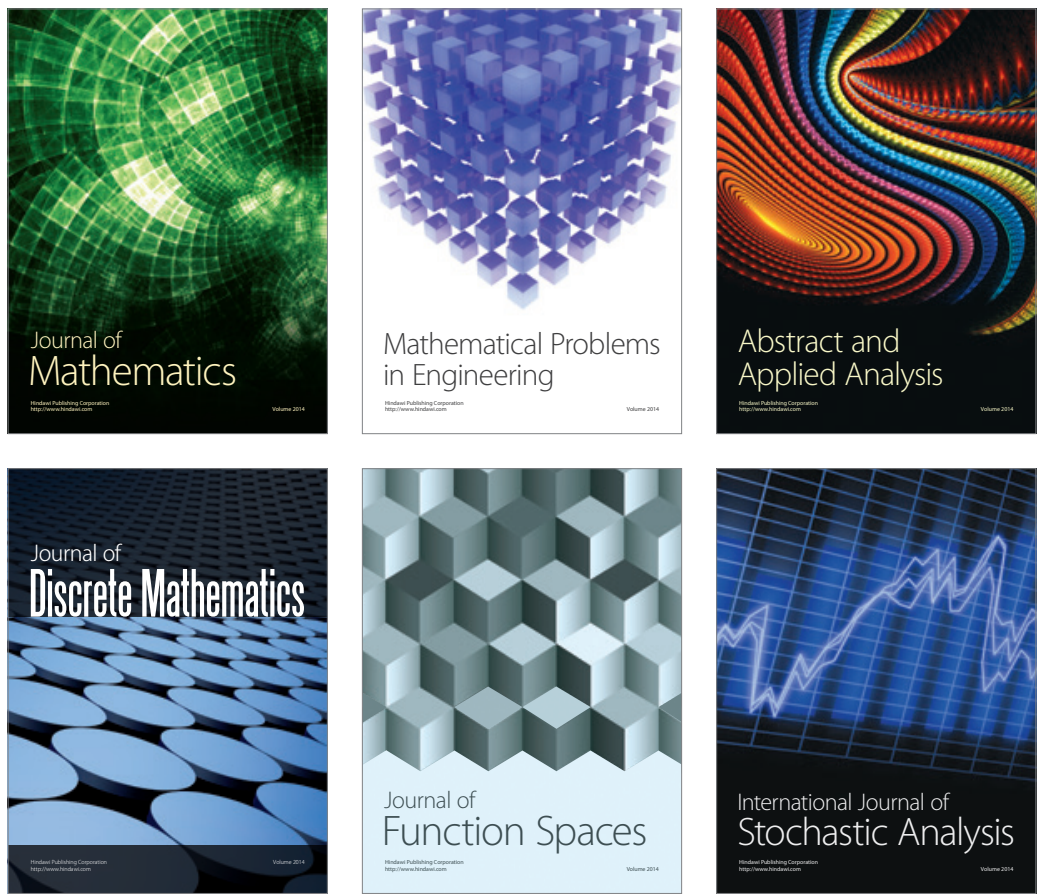

Journal of

Function Spaces

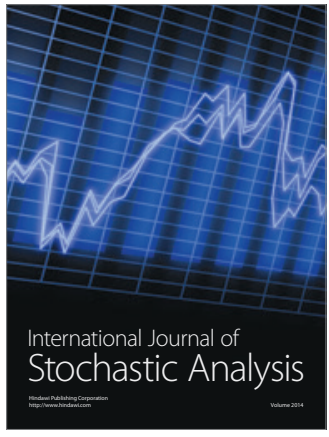

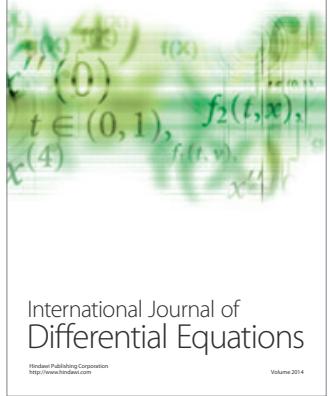
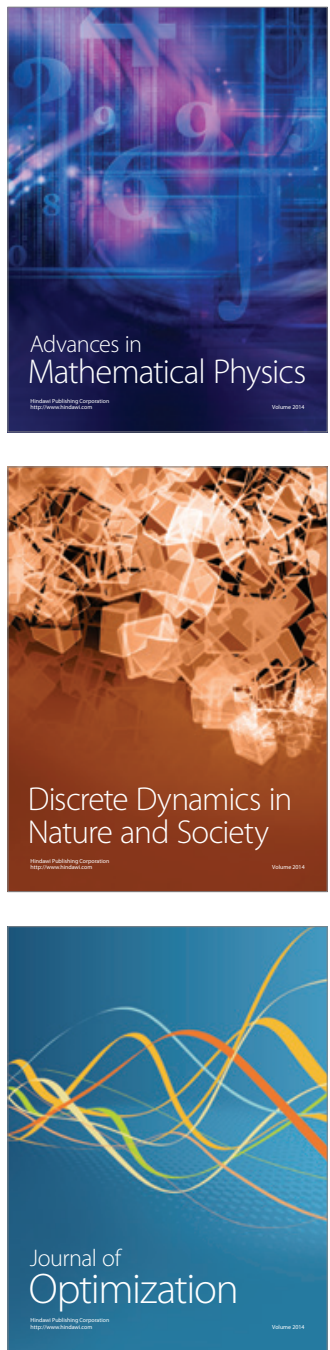